\title{
miR-519d-3p Inhibits Cell Proliferation and Invasion of Gastric Cancer by Downregulating B-Cell Lymphoma 6
}

\author{
Yong-Yuan $\mathrm{Li}^{\mathrm{a}}$ Jian-Ping Shao ${ }^{\mathrm{a}}$ Shu-Peng Zhang ${ }^{\mathrm{a}}$ Guo-Qiang Xing ${ }^{\mathrm{a}}$ \\ Hong-Jie Liu ${ }^{\text {b }}$ \\ Departments of a General Surgery and ${ }^{b}$ Radiology, The Fifth Central Hospital of Tianjin, Tianjin, PR China
}

\section{Keywords}

BCL6 · Cell cycle · Gastric cancer · miR-519d-3p · miRNA

\begin{abstract}
miR-519d inhibits cell growth, migration, and invasion, but its role in gastric cancer (GC) cells is obscure. We showed that miR-519d-3p was lowly expressed in GC tissues and was associated with the clinical stage and lymph node metastasis of GC tissues. We found that miR-519d-3p repressed cell proliferation and invasion of MGC803 cells and delayed the G1/S phase transition, resulting in decreased cyclin B1 and MMP2 and increased E-cadherin levels. Furthermore, miR-519d-3p targeted and downregulated B-cell lymphoma 6 (BCL6) expression. BCL6 overexpression partially abrogated the suppressive function of miR-519d in MGC803 cells. In conclusion, our study demonstrated that miR-519d-3p functions as a tumor suppressor by targeting and downregulating the expression of BCL6 in GC cells.

(C) 2018 The Author(s) Published by S. Karger AG, Basel
\end{abstract}

Gastric cancer (GC) is the third most common cancer worldwide [Ferlay et al., 2015]. Although increasingly effective treatments for GC have been developed, the prog-

\begin{tabular}{ll}
\hline KARGER & $\begin{array}{l}\text { ○ } 2018 \text { The Author(s) } \\
\text { Published by S. Karger AG, Basel Oprger }\end{array}$ \\
$\begin{array}{l}\text { E-Mail karger@karger.com } \\
\text { www.karger.com/cgr }\end{array}$ & $\begin{array}{l}\text { This article is licensed under the Creative Commons Attribution- } \\
\text { NonCommercial-NoDerivatives } 4.0 \text { International License (CC BY- } \\
\text { NC-ND) (http://www.karger.com/Services/OpenAccessLicense). } \\
\text { Usage and distribution for commercial purposes as well as any dis- } \\
\text { tribution of modified material requires written permission. }\end{array}$
\end{tabular}

nosis of GC remains poor [Shimizu et al., 2018]. The persistent poor prognosis therefore motivated the search for further optimization strategies for GC prevention and treatment.

microRNA (miRNA) is a class of 21-24 nucleotide noncoding small RNAs that bind to the $3^{\prime}$-untranslated region ( $3^{\prime}$-UTR) of the messenger RNA to regulate the expression of target genes [Bartel, 2004]. miRNAs play a vital role in various biological processes, including cell differentiation, growth, migration, and invasion [Lujambio and Lowe, 2012; Sun and Lai, 2013]. miR-519d-3p participates in the development of numerous cancers, such as ovarian cancer, suppressing cell proliferation by downregulating XIAP [Pang et al., 2014]. It also targets MKi67, represses the growth of hepatocellular carcinoma cells [Hou et al., 2011], and inhibits the malignant phenotype of colorectal cancer by targeting C14orf28 expression [Yang et al., 2017]. Moreover, miR-519d-3p may act as an oncogene and promote the progression of cervical cancer [Zhou et al., 2016]. However, the function of miR$519 \mathrm{~d}-3 \mathrm{p}$ in $\mathrm{GC}$ is unclear.

Here, we demonstrated that miR-519d-3p repressed cell proliferation and invasion of MGC803 cells. In addition, miR-519d-3p delayed G1/S phase transition, resulting in decreased cyclin B1 and MMP2 and increased E-cadherin

Yong-Yuan Li

Department of General Surgery

The Fifth Central Hospital of Tianjin

41 Tanggu Zhejiang Road, Binhai New Area, Tianjin 300450 (PR China)

E-Mail wzxlyy@hotmail.com 
levels. B-cell lymphoma 6 (BCL6) is the target of miR$519 \mathrm{~d}-3 \mathrm{p}$, and its ectopic expression restored the inhibitory effect of miR-519d-3p on cell proliferation and invasion in MGC803 cells. miR-519d-3p was downregulated whereas BCL6 was upregulated in human GC tissues and cell lines. Our findings can provide insight into the development of GC by altering the regulation of miRNA pathways.

\section{Materials and Methods}

Cell Culture and Transfection

GES-1 (DMEM), AGS (F-12K), SCG-7901(RPMI 1640), BGC823 (DMEM), and MGC803 cells (RPMI 1640) were cultured and propagated in the respective medium with $10 \%$ fetal bovine serum. All transfections were performed according to the manufacturer's protocol with LipofectAmine 2000 reagent.

\section{Vector Construction}

The miR-519d-3p mimics and inhibitor and the BCL6 overexpression plasmid, $3^{\prime}$-UTR of BCL6 and $3^{\prime}$-UTR-mutant of BCL6 gene expression plasmids were obtained from Invitrogen (Carlsbad, CA, USA).

\section{$R T-q P C R$}

Total RNA was extracted with TRIzol reagent (Sigma-Aldrich) and reversely transcribed into cDNA using random or special primers. The special primers used for reversely transcribed included were miR-519d-3p-RT, 5'-GTCGTATCCAGTGCAGGG TCCGAGGTGCACTGGATACGACCACTCTA-3'; U6-RT, 5'-GTCGTATCCAGT GCAGGGTCCGAGGTGCACTGGATACGACAAAATATGG-3'. RT-qPCR was done with the following primers: miR-519d-3p sense, $5^{\prime}$-TGCGGCAAAGTGCCTCCCTTTAG-3', miR-519d-3p antisense, 5'-CCAGTGCAGGGTCCGAG GT-3'; U6 sense, 5'-TGCGGGTGCTCGCTTCGGCAGC-3', U6 antisense, 5' -CCAGTGCAGGGTCCGAGGT-3'. BCL6 sense, 5' -TCCTCGGAAGATGAGATTGC-3', BCL6 antisense, 5'-GTTGAGCACGATGAACTTGTA- $3^{\prime} ; \quad \beta$-actin sense, $5^{\prime}$-CTACGTCGCCCTGGACTTCGAGC- ${ }^{\prime}$; and $\beta$-actin antisense, $5^{\prime}$ - GATGGAGCCGCCGATCCACACGG-3'. The details of the experimental protocol of RNA extraction and RT-qPCR can be found in Wan et al. [2014].

Western Blot Assay

Protein extractions and the experimental procedure of Western blot were performed as described in Hou et al. [2011]. AntiBCL6, anti-cyclin B1, anti-E-cadherin, anti-MMP2, and antiGAPDH were from Abcam (Cambridge, UK).

\section{Luciferase Reporter Assay}

The MGC803 cells were transiently transfected with miR519d-3p mimics, miR-control, ASO-miR-519d-3p, ASO-control, pLuc-BCL6-3' UTR, and pLuc-BCL6-3' UTR-mut in 48-well plates. The $\mathrm{pRL}-\mathrm{TK}$ was included as a transfection efficiency control [Wang et al., 2016a].

MTT, Colony Formation, and Invasion Assays

Details of the procedure of MTT, colony formation, and invasion assays can be found in Long et al. [2012] and Wan et al. [2014].

miR-519d-3p Downregulates BCL6
Cell Cycle Analysis

Transfected MGC803 cells were plated in 6-well plates and incubated for $72 \mathrm{~h}$ in complete culture medium. Cell cycle analysis by flow cytometry was performed following the method described by Zhao et al. [2015].

\section{Statistical Analysis}

All statistical analyses were performed with 2-tailed unpaired Student's $t$ test to make the comparison, and $p \leq 0.05$ was considered statistically significant. The graphs represent the average results of 3 experiments.

\section{Results}

miR-519d-3p Inhibits a Malignant Phenotype and Arrests G1/S Phase Transition in GC Cells

To investigate whether miR-519d-3p affects GC cell growth, we transfected MGC803 cells with miR-519d-3p mimics, ASO-miR-519d-3p, or a negative control. The efficiency of the vectors was validated by RT-qPCR prior to further analyses (Fig. 1A). Thus, MTT and colony formation assays were performed in MGC803 cells. The overexpression of miR-519d-3p by transfection with miR-519d-3p mimics inhibited the proliferation of MGC803 cells, whereas decreased levels of miR-519d-3p expression displayed opposite effects (Fig. 1B, C). We subsequently detected cell cycle progression by flow cytometry analysis. As shown in Figure 1D, miR-519d-3p increased the number of cells in G1 phase but decreased the cells in S phase relative to the negative control. A Transwell assay showed that MGC803 cell invasion capacity was repressed by miR-519d-3p overexpression and facilitated by miR-519d-3p inhibition (Fig. 1E). These results showed that miR-519d-3p inhibited MGC803 cell proliferation and invasion and delayed $\mathrm{G} 1 / \mathrm{S}$ phase transition.

\section{BCL6 Is the Target of miR-519d-3p}

To determine target genes that mediate the function of miR-519d-3p in GC, we used bioinformatic analysis algorithms MIRDB, RNAhybrid, and TargetScan to predict candidate targets of miR-519d-3p. On the basis of the analysis of functions among targets, we selected BCL6 as a candidate. To validate whether BCL6 is targeted by miR519d-3p, we constructed luciferase reporter plasmids carrying the $3^{\prime}$-UTR of a BCL6 fragment or the mutant sites of the miR-519d-3p targeting site (Fig. 2A). The luciferase reporter assay showed that compared with the control group, miR-519d-3p overexpression and inhibition, respectively, decreased the BCL6 $3^{\prime}$-UTR fluorescence in-

Cytogenet Genome Res 2018;154:12-19 


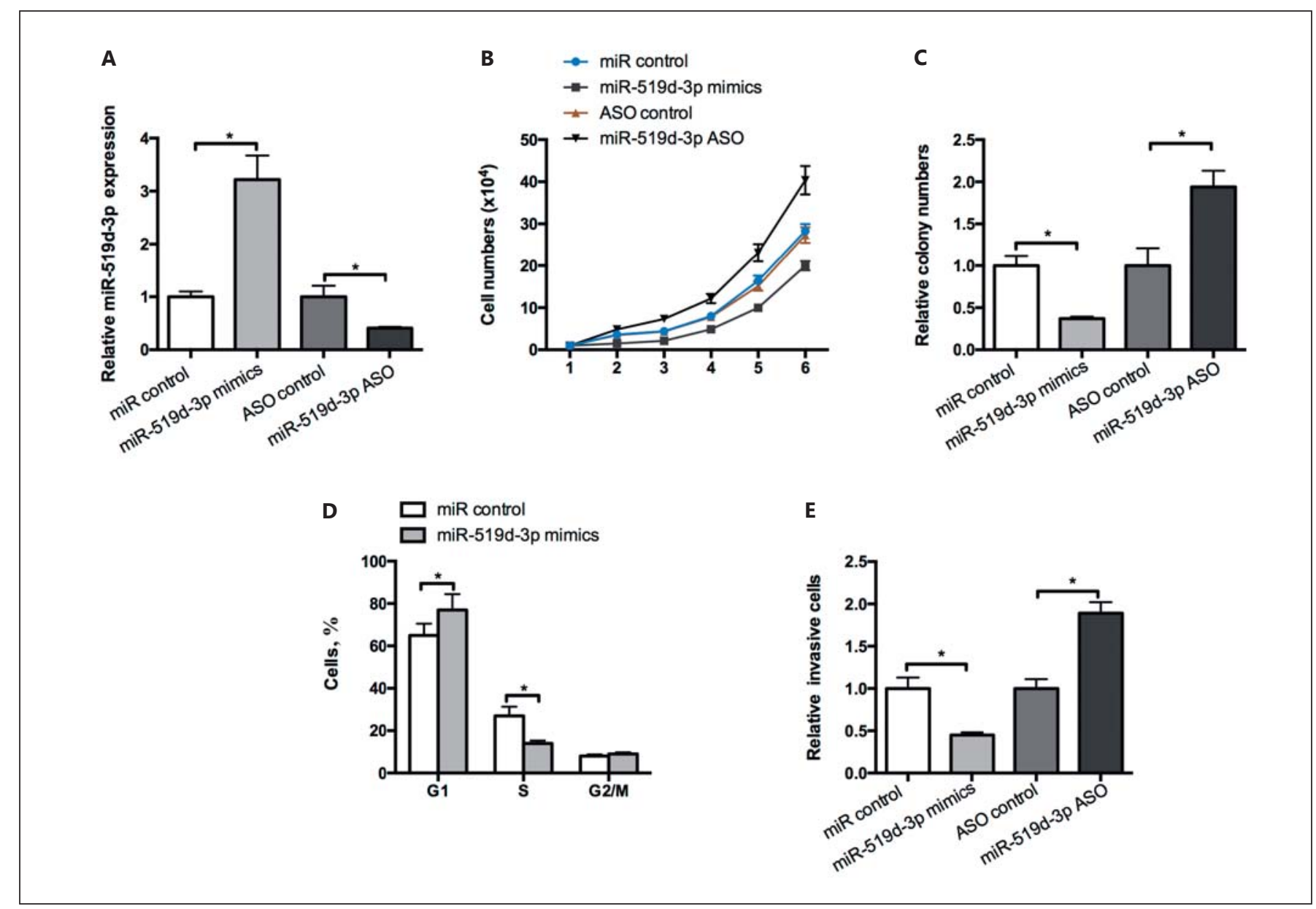

Fig. 1. miR-519d-3p inhibits the malignant phenotype and arrests G1/S phase transition in GC cells. A An RT-qPCR assay was used to test the efficiency of miR-519d-3p mimics and ASO-miR-519d3 p in MGC803 cells. MTT (B) and colony formation assays (C) were performed to test the effect of miR-519d-3p on MGC803 cell pro- liferation. D The effect of miR-519d-3p on the cell cycle in MGC803 cells was analyzed by flow cytometry. E Transwell invasion assays were conducted in MGC803 cells transfected with miR-519d-3p mimics and ASO-miR-519d-3p, and miR control or ASO control were considered as the corresponding negative controls. ${ }^{*} p<0.05$. tensity of MGC803 cells. By contrast, neither miR-519d$3 p$ overexpression nor inhibition altered the fluorescence intensity of BCL6-3'-UTR-mut (Fig. 2B). We examined the regulatory role of miR-519d-3p on endogenous BCL6 in MGC803 cells. RT-qPCR and Western blot assay were conducted to detect the mRNA and protein levels of BCL6, respectively. As shown in Figure 2C and D, the RNA and protein expression levels of BCL6 were reduced by the overexpression of miR-519d-3p. These findings indicate that miR-519d-3p targets the $3^{\prime}$-UTR of BCL6 and negatively regulates BCL6 expression.

\section{miR-519d-3p/BCL6 Axis Regulates a Malignant \\ Phenotype in GC Cells}

We performed a series of rescue experiments to demonstrate that the effect of miR-519d-3p on MGC803 cells was mediated by regulating BCL6. Western blot assay showed that BCL6 overexpression restored the decreased BCL6 protein levels caused by miR-519d-3p (Fig. 3A). Moreover, functional rescue experiments showed that the miR-519d-3p-mediated suppression of colony formation in the MGC803 cell was counteracted by the ectopic expression of BCL6 (Fig. 3B). In addition, the restoration of BCL6 expression mainly reestablished the inhibitory effect on the invasion capability caused by miR-519d-3p
Li/Shao/Zhang/Xing/Liu 


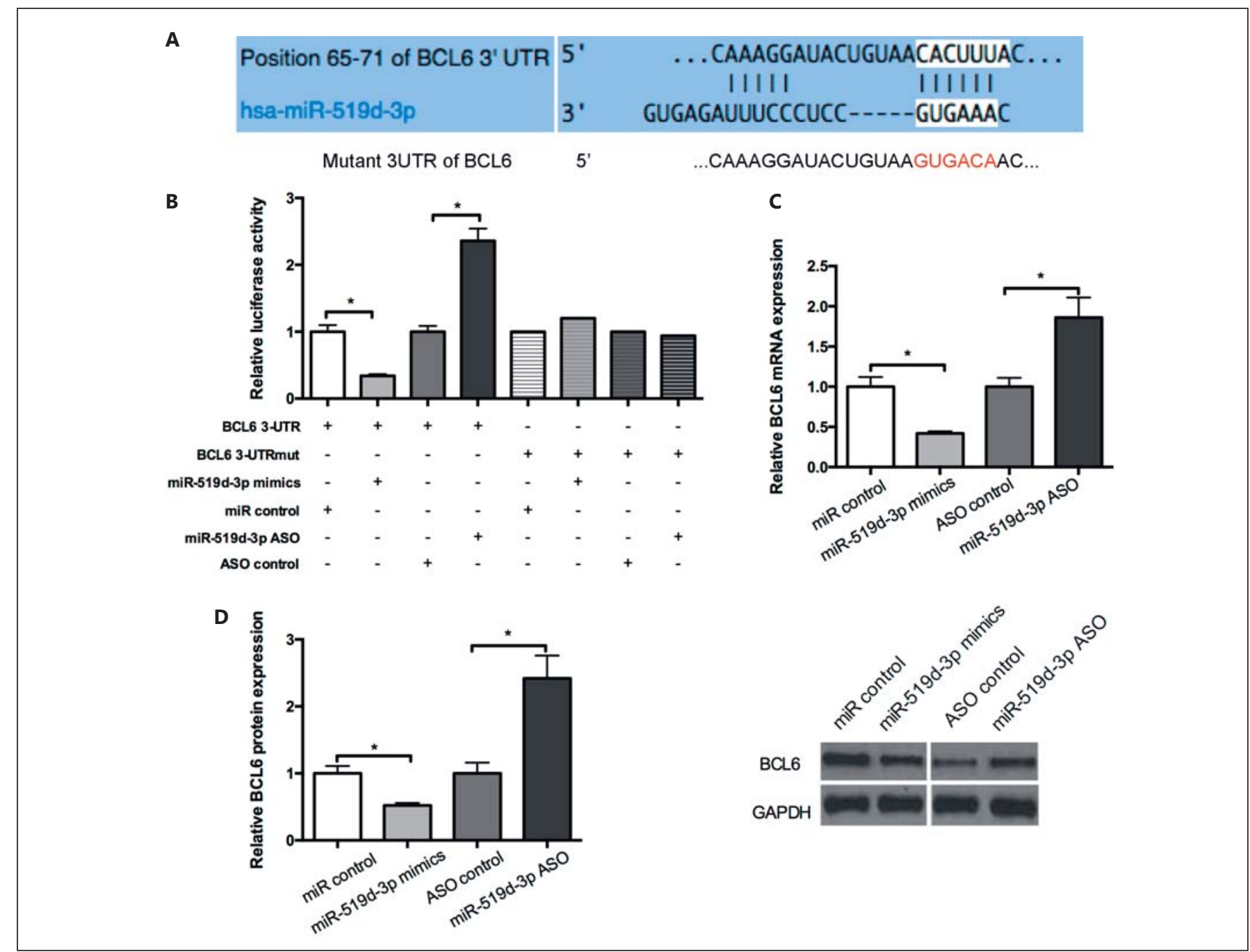

Fig. 2. miR-519d-3p targets BCL6. A The predicted potential binding site of miR-519d-3p at the $3^{\prime}$-UTR of BCL6 and the mutant $3^{\prime}$-UTR of BCL6 is shown. B A luciferase reporter assay was performed in MGC803 cells co-transfected with miR-519d-3p mimics and ASO-miR-519d-3p or control vector with $3^{\prime}$-UTR or $3^{\prime}$-UTR-

(Fig. 3C). As shown in Figure 3D, compared with the negative control, BCL6 restored the increase in the number of cells in G1 phase and a decrease in the number of cells in $S$ phase caused by miR-519d-3p. These results indicate that BCL6 is a mediator of miR-519d-3p-inhibited GC cell proliferation, cell cycle, and invasive capacity.

miR-519d-3p/BCL6 Axis Regulates Molecule Makers of Cell Cycle and Endothelial-Mesenchymal Transition

To investigate the underlying mechanism of the inhibition of cell proliferation, invasion, and cell cycle by miR-

miR-519d-3p Downregulates BCL6 mut of BCL6. The fluorescence intensity was measured $48 \mathrm{~h}$ post transfection. C, D MGC803 cells transfected with miR-519d-3p mimics and ASO-miR-519d-3p or control vector. At $48 \mathrm{~h}$ posttransfection, BCL6 mRNA and protein levels were determined using RT-qPCR and Western blot, respectively. $* p<0.05$.

$519 d-3 p$, we conducted a Western blot assay to detect certain molecular markers of the cell cycle and the endothelial-mesenchymal transition (EMT). As shown in Figure 4, compared with the negative control, miR-519d-3p overexpression decreased the levels of cyclin $\mathrm{B} 1$ protein and MMP2 and increased the E-cadherin level. In addition, the restoration of BCL6 expression counteracted the reduction effect on cyclin B1, E-cadherin, and MMP2 protein levels by miR-519d-3p. Taken together, these data indicate that the miR-519d-3p/BCL6 axis inhibits cell proliferation, invasion, and cell cycle by regulating cyclin B1, E-cadherin, and MMP2 levels in GC cells. 


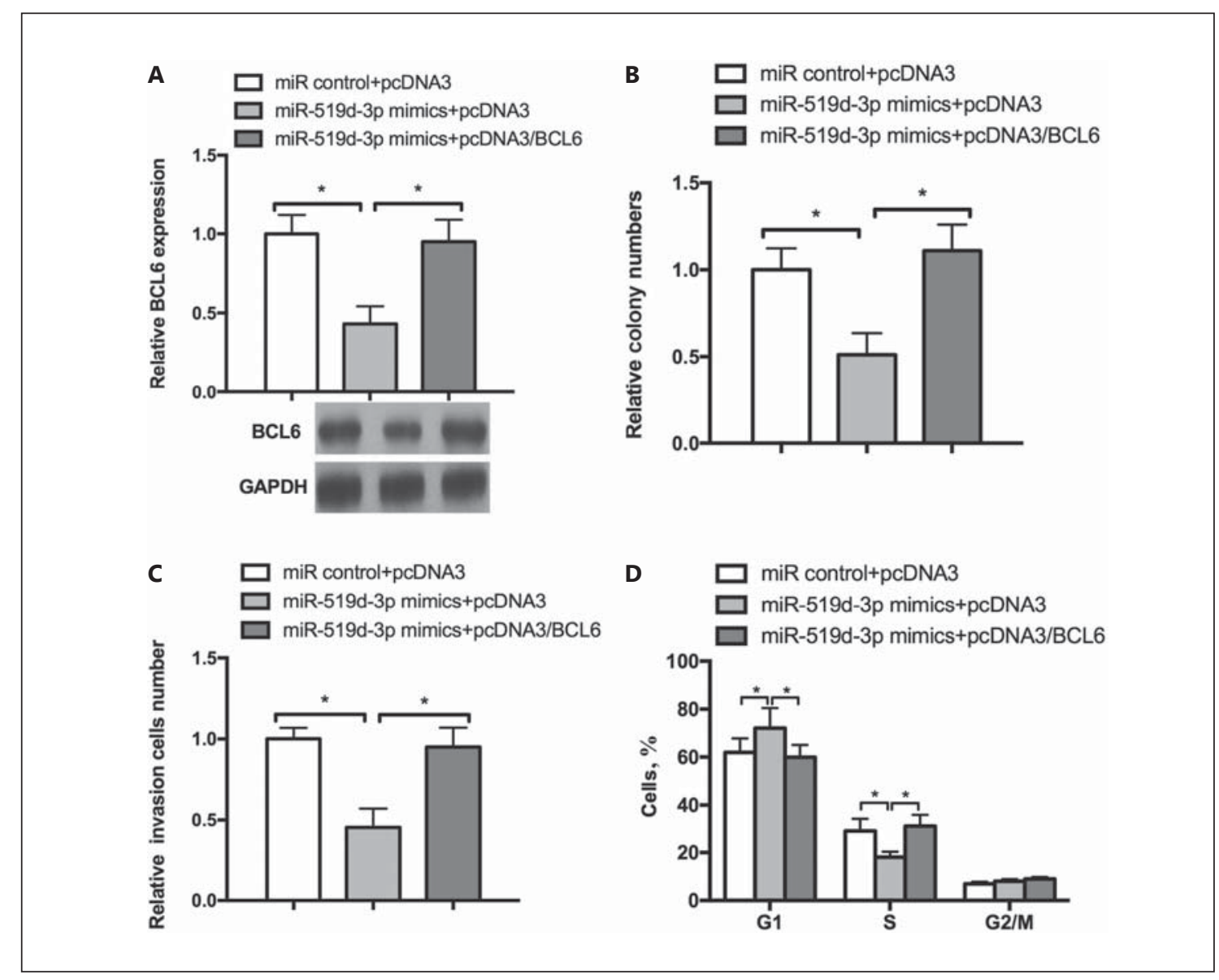

Fig. 3. miR-519d-3p/BCL6 axis regulates a malignant phenotype in GC cells. A MGC803 cells were cotransfected with miR-519d-3p mimics and pcDNA3/BCL6 or the control vector. Western blot was performed to determine the BCL6 protein level. B-D The transfected cells were submitted to detect the colony formation rate (B), invasive ability (C), and cell cycle (D). ${ }^{*} p<0.05$.

miR-519d-3p Level Is Inversely Correlated with

BCL6 in GC Tissues and Cells and Related to the

Clinicopathologic Characteristics of GC Patients

Considering that the miR-519d-3p/BCL6 axis regulates the malignant phenotype of GC cells, we aimed to analyze the expression of miR-519d-3p and BCL6 in patients with GC. RT-qPCR analysis was carried out to detect miR-519d-3p and BCL6 levels in 20 paired GC tumor tissue samples. As shown in Figure 5A, in contrast with adjacent nontumor tissues, miR-519d-3p and BCL6 levels were downregulated and upregulated in GC tumor tissues, respectively. The association between miR-519d-3p and the clinicopathologic characteristics of GC tumor tissues was analyzed, and it was found that miR-519d-3p was associated with the clinical stage and lymph node metastasis of GC tissues. Decreased miR-519d-3p expression was associated with advanced stage and lymph node me- tastasis of GC compared with controls (Fig. 5B). Then, we detected miR-519d-3p and BCL6 levels in several GC cell lines by RT-qPCR. Compared with gastric primary cells (GES-1), miR-519d-3p was downregulated and BCL6 was upregulated in GC cell lines, including AGS, SCG-7901, BGC-823, and MGC803 cells (Fig. 5C). These results indicated that miR-519d-3p was downregulated, whereas BCL6 was upregulated in GC tissues and cell lines and correlated with a malignant phenotype in patients with GC.

\section{Discussion}

miRNAs play a vital role in regulating cellular biological processes, and their aberrant expression is related to cancer progression. In GC, the dysregulation of the expression of miRNAs, including miR-106a [Zhu et al.,
Li/Shao/Zhang/Xing/Liu 
Fig. 4. miR-519d-3p/BCL6 axis regulates the molecular markers of the cell cycle and the endothelial-mesenchymal transition. MGC803 cells were cotransfected with miR-519d-3p mimics and pcDNA3/BCL6 or the control vector. Western blot was used to assay the protein levels of cyclin B1, E-cadherin, and MMP2.

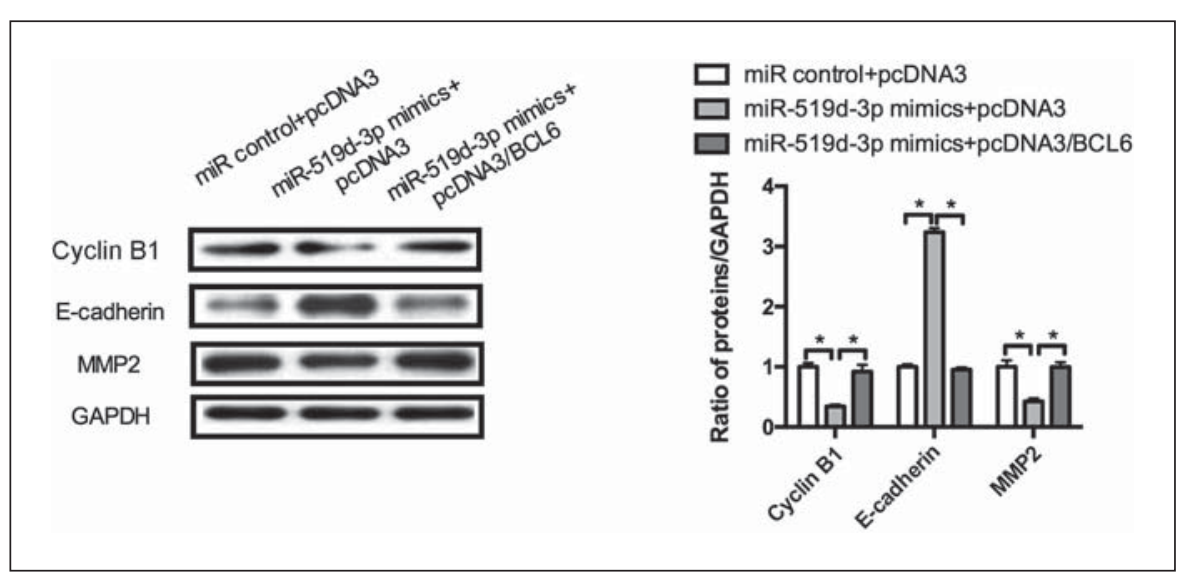

C

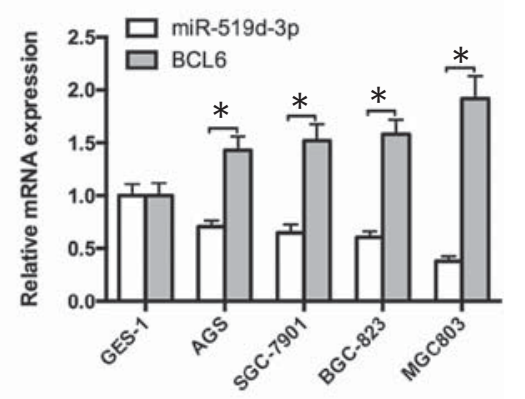

Fig. 5. miR-519d-3p level is inversely correlated with BCL6 in GC tissues and cells and related to the clinicopathologic characteristics of patients with GC. A RT-qPCR was conducted to test the relative expression level of miR-519d-3p and BCL6 in 20 paired GC tissues and adjacent nontumor tissues. U6 snRNA was used

2014], let-7a [Yang et al., 2011], miR-107 [Wang et al., 2016b], and miR-181b [Guo et al., 2012], is an important regulator of biological cellular processes. Here, we first revealed that miR-519d-3p was downregulated in human GC tissues in contrast with the adjacent nontumor tissues, and depressed miR-519d expression was associated with the more advanced stage and lymph node metastasis of GC. Functional analyses showed that miR-519d-3p inhibited cell proliferation and MGC803 cell invasion. Moreover, miR-519d-3p delayed the G1/S phase transition, resulting in decreased cyclin B1 and MMP2 and increased E-cadherin levels. E-cadherin is an important molecular marker of EMT, which is a vital driver of cancer metastasis [Gomes et al., 2011; Yeung and Yang,

miR-519d-3p Downregulates BCL6 as an internal control. B Correlation between miR-519d-3p and the clinicopathologic characteristics of patients with GC. C The relative expression levels of miR-519d-3p and BCL6 were detected in GES-1, AGS, SCG-7901, BGC-823, and MGC803 cell lines by RT-qPCR.

2017]. MMP2 can degrade a variety of extracellular matrices and plays a vital part in invasion [Xiang et al., 2011; Wang et al., 2014]. miR-519d-3p inhibits cell invasion by regulating the expression of E-cadherin and MMP2.

miRNA functions by downregulating the expression of its target gene [Bartel, 2009]. We used bioinformatics assays (MIRDB, RNAhybrid, and TargetScan) to predict and identify BCL6 as a new target of miR-519d-3p. We performed a luciferase reporter assay to show that miR519d-3p negatively regulated the BCL6 3'-UTR fluorescence intensity. RT-qPCR assay indicated that miR-519d$3 p$ repressed endogenous BCL6 expression at the mRNA and protein level. In addition, an inverse relationship was observed between miR-519d-3p and BCL6 in GC tissues.

Cytogenet Genome Res 2018;154:12-19 
Accordingly, BCL6 was targeted and downregulated by miR-519d-3p.

BCL6 belongs to the BTB-POZ family and was originally identified as a protooncogene that acts as a transcriptional factor [Ohno, 2006]. BCL6 facilitates the cell phenotype by repressing genes that control cell death, cell cycle arrest, DNA damage response, and cell differentiation in the germinal center [Shaffer et al., 2000; Phan and Dalla-Favera, 2004; Phan et al., 2005; Ranuncolo et al., 2007, 2008]. Here, we demonstrated that BCL6 was upregulated in human GC tissues compared with adjacent nontumor tissues. The miR-519d-3p-mediated suppression of cell proliferation, cell cycle, and invasive capacity in MGC803 cells was counteracted by ectopic expression of BCL6. Moreover, the restoration of BCL6 expression counteracted the reduction effect on cyclin B1, E-cadherin, and MMP2 proteins by miR-519d-3p. Taken together, our results show that the miR-519d-3p/BCL6 axis inhibits cell proliferation, invasion, and cell cycle by regulating cyclin B1, E-cadherin, and MMP2 levels in GC cells.
In summary, our findings demonstrate that miR$519 \mathrm{~d}-3 \mathrm{p}$ targets the $3^{\prime}$-UTR of BCL6, downregulating its expression. BCL6 mediates the repression of miR-519d$3 p$ on cell proliferation and invasive capability of GC cells. These findings may provide new ideas for understanding GC progression and may promote the development of preventive and therapeutic strategies for GC.

\section{Statement of Ethics}

The research involving human subjects was ethically conducted in accordance with the World Medical Association Declaration of Helsinki. The patients (or their parents or guardians) have given their informed written consent, and the study protocol was approved by an appropriate ethics committee. All patients were identified by numbers or aliases, not by their real names.

\section{Disclosure Statement}

The authors have no conflict of interest to declare.

\section{References}

Bartel DP: MicroRNAs: genomics, biogenesis, mechanism, and function. Cell 116:281-297 (2004).

Bartel DP: MicroRNAs: target recognition and regulatory functions. Cell 136:215-233 (2009).

Ferlay J, Soerjomataram I, Dikshit R, Eser S, Mathers C, et al: Cancer incidence and mortality worldwide: sources, methods and major patterns in GLOBOCAN 2012. Int J Cancer 136:E359-386 (2015).

Gomes LR, Terra LF, Sogayar MC, Labriola L: Epithelial-mesenchymal transition: implications in cancer progression and metastasis. Curr Pharm Biotechnol 12:1881-1890 (2011).

Guo JX, Tao QS, Lou PR, Chen XC, Chen J, Yuan GB: miR-181b as a potential molecular target for anticancer therapy of gastric neoplasms. Asian Pac J Cancer Prev 13:2263-2267 (2012).

Hou YY, Cao WW, Li L, Li SP, Liu T, et al: MicroRNA-519d targets MKi67 and suppresses cell growth in the hepatocellular carcinoma cell line QGY-7703. Cancer Lett 307:182-190 (2011).

Long MJ, Wu FX, Li P, Liu M, Li X, Tang H: MicroRNA-10a targets CHL1 and promotes cell growth, migration and invasion in human cervical cancer cells. Cancer Lett 324:186-196 (2012).

Lujambio A, Lowe SW: The microcosmos of cancer. Nature 482:347-355 (2012).
Ohno H: Pathogenetic and clinical implications of non-immunoglobulin; BCL6 translocations in B-cell non-Hodgkin's lymphoma. J Clin Exp Hematopathol 46:43-53 (2006).

Pang Y, Mao H, Shen L, Zhao Z, Liu R, Liu P: MiR-519d represses ovarian cancer cell proliferation and enhances cisplatin-mediated cytotoxicity in vitro by targeting XIAP. Onco Targets Ther 7:587-597 (2014).

Phan RT, Dalla-Favera R: The BCL6 proto-oncogene suppresses p53 expression in germinalcenter B cells. Nature 432:635-639 (2004).

Phan RT, Saito M, Basso K, Niu H, Dalla-Favera R: BCL6 interacts with the transcription factor Miz-1 to suppress the cyclin-dependent kinase inhibitor p21 and cell cycle arrest in germinal center B cells. Nat Immunol 6:10541060 (2005).

Ranuncolo SM, Polo JM, Dierov J, Singer M, Kuo $\mathrm{T}$, et al: Bcl-6 mediates the germinal center B cell phenotype and lymphomagenesis through transcriptional repression of the DNA-damage sensor ATR. Nat Immunol 8: 705-714 (2007).

Ranuncolo SM, Polo JM, Melnick A: BCL6 represses CHEK1 and suppresses DNA damage pathways in normal and malignant B-cells. Blood Cells Mol Dis 41:95-99 (2008).

Shaffer AL, Yu X, He Y, Boldrick J, Chan EP, Staudt LM: BCL-6 represses genes that function in lymphocyte differentiation, inflammation, and cell cycle control. Immunity 13:199_ 212 (2000).
Shimizu D, Kanda M, Kodera Y: Review of recent molecular landscape knowledge of gastric cancer. Histol Histopathol 33:11-26 (2018).

Sun K, Lai EC: Adult-specific functions of animal microRNAs. Nat Rev Genet 14:535-548 (2013).

Wan HY, Li QQ, Zhang Y, Tian W, Li YN, et al: MiR-124 represses vasculogenic mimicry and cell motility by targeting amotL1 in cervical cancer cells. Cancer Lett 355:148-158 (2014).

Wang B, Ding YM, Fan P, Wang B, Xu JH, Wang WX: Expression and significance of MMP2 and HIF-1alpha in hepatocellular carcinoma. Oncology Lett 8:539-546 (2014).

Wang Y, Sun B, Zhao X, Zhao N, Sun R, et al: Twist1-related miR-26b-5p suppresses epithelial-mesenchymal transition, migration and invasion by targeting SMAD1 in hepatocellular carcinoma. Oncotarget 7:2438324401 (2016a).

Wang S, Ma G, Zhu H, Lv C, Chu H, et al: miR-107 regulates tumor progression by targeting NF1 in gastric cancer. Sci Rep 6:36531 (2016b).

Xiang ZL, Zeng ZC, Fan J, Tang ZY, Zeng HY, Gao DM: Gene expression profiling of fixed tissues identified hypoxia-inducible factor1alpha, VEGF, and matrix metalloproteinase- 2 as biomarkers of lymph node metastasis in hepatocellular carcinoma. Clin Cancer Res 17:5463-5472 (2011) 
Yang Q, Jie Z, Cao H, Greenlee AR, Yang C, et al: Low-level expression of let-7a in gastric cancer and its involvement in tumorigenesis by targeting RAB40C. Carcinogenesis 32:713722 (2011).

Yang X, Hu Y, Liu Y, Liu W, Zhao X, et al: C14orf28 downregulated by miR-519d contributes to oncogenicity and regulates apoptosis and EMT in colorectal cancer. Mol Cell Biochem 434:197-208 (2017).
Yeung KT, Yang J: Epithelial-mesenchymal transition in tumor metastasis. Mol Oncol 11:2839 (2017).

Zhao JL, Zhang L, Guo X, Wang JH, Zhou W, et al: miR-212/132 downregulates SMAD2 expression to suppress the $\mathrm{G} 1 / \mathrm{S}$ phase transition of the cell cycle and the epithelial to mesenchymal transition in cervical cancer cells. IUBMB Life 67:380-394 (2015).
Zhou JY, Zheng SR, Liu J, Shi R, Yu HL, Wei M: miR-519d facilitates the progression and metastasis of cervical cancer through direct targeting Smad7. Cancer Cell Int 16:21 (2016).

Zhu M, Zhang N, He S, Lui Y, Lu G, Zhao L: MicroRNA-106a targets TIMP2 to regulate invasion and metastasis of gastric cancer. FEBS Lett 588:600-607 (2014). 